\title{
Intonational strategies for backchanneling in Italian Map Task dialogues
}

\author{
Michelina Savino \\ Department of Psychology, University of Bari, Italy \\ IfL-Phonetics, University of Cologne, Germany \\ https://doi.org/10.36505/ExLing-2010/03/0040/000160
}

\begin{abstract}
This paper investigates intonational strategies for backchanneling in Bari Italian task-oriented dialogues. Results show that whilst giving feedback, listeners indicate their intention to take the floor or not by using a falling or rising intonation contour respectively. However, this general tendency can be reversed if added paralinguistic meanings are conveyed.
\end{abstract}

Keywords: Backchannels, Prosody, Italian language, Map Task dialogues

\section{Introduction}

The crucial role played by "acknowledge tokens" or "continuers" (Schegloff 1982, Jefferson 1983) in verbal communication is widely recognised. These include lexical and non-lexical tokens (like 'yes', 'yeah', 'mm', 'uh uh', ect) which can be used for backchanneling, i.e. for signalling that the listener is attending to the speaker and prompting her/him to go on. Past research work on a number of languages has preliminary ascertained that continuers are generally characterised by a rising contour as opposed, for example, to yes-reply tokens which normally have a falling F0 shape (for example Jurafky et al 1998, Caspers 2000, Cerrato and D'Imperio 2003, Benus et al 2007). The aim of this study is to contribute to investigating the role of prosody in the pragmatic interpretation of backchanneling in Italian.

\section{Materials and method}

Analysed speech data consist of 5 Bari Italian dialogues elicited with a modified version of the Map Task methodology (Carletta et al. 1997), in that participants were not informed about discrepancies between the two maps (how this feature plays a role in speakers' intonational strategies of backchanneling is discussed later). Dialogues were pragmatically analysed using both orthographic transcriptions and audio files. All "acknowledge" and "positive reply" tokens realised with 'sì' (yes), 'mm', 'eh', and 'okay' were selected and intonationally analysed with Praat. For the pragmatic annotation of backchannels, an additional criterion with respect to the broad category of "Acknowledge" move proposed in the Map Task coding scheme (Carletta et al. 1997) was adopted, in which turn-taking dynamics was taken into account. Following Jurafky et al. (1998) work (inspired by theoretical assumptions in

ExLing 2010: Proceedings of 3rd Tutorial and Research Workshop on Experimental Linguistics, 25-27 August, Athens, Greece 
Jefferson 1983), we distinguished continuers reflecting "Passive Recipiency" (henceforth PR), i.e. those acknowledging the other speaker still holds the turn, from continuers signalling "Incipient Speakership" (henceforth IS), i.e. indicating the intention of taking the floor. Decisions on whether an acknowledge token was to be classified as an example of PR or IS were based on whether or not a change of speaker was involved immediately after the token. In order to avoid possible circularity in the pragmatic interpretation of backchannels, our initial analysis relied on this one criterion. This constitutes a sort of "bottom-up" approach, where a finer pragmatic distinction of backchanneling subcategories can be derived $a$ posteriori by combining analysis of prosody and of communicative context. In order to compare the intonational realisations of backchannels with the same token types used as positive replies, we also analysed all target tokens in "Reply-Y" moves, i.e. positive replies to any yes-no questions as realised in "Query-YN", "Check" and "Align" moves (Grice \& Savino 2003).

\section{Results}

Continuers marking PR are mainly realised with the lexical token 'sì', followed by 'mm' and 'okay' (Figure 2, left panel). These continuers are intonationally characterised predominantly by a rising pitch, where a wide range of contour choices is available: rise, fall-rise, "stylised rise", and also high rise. The most widespread F0 pattern is the falling-rising (Figure 2, right panel): in the dialogues, this is typically used by Instruction Followers (IFs) for conveying not only understanding and agreement about the instructions being received, but also for signalling they are compatible with the presence and position of landmarks on her/his map, i.e. for confirming the current status of shared background knowledge. The stylised rise is only found in ' $\mathrm{mm}$ ' tokens, and in such cases they are produced as bisyllabic, as also observed in English backchannels (Ward 2004). The stylised rise is realised as a pitch stepup from the first to the second syllable, where the pitch excursion is not wide. Examples of these 3 contour types in 'mm' are shown in Figure 1. A number of high rising contours are also found, typically used by IFs for conveying her/his understanding and agreement that the end of a (sub)set of instructions for completing a (sub)task is approaching. This contour has already been described in Bari Italian for signalling pre-finality (i.e. marking the antepenultimate item in a sequence) and phonologically analysed with $\mathrm{H}^{*} \mathrm{H}-\mathrm{H} \%$ (Savino 2001;2004). Even though backchannels marking PR are generally characterised by a high pitch, we found a large number of these continuers as realised with a falling (or sometimes flat) F0 contour instead. This pattern is usually encountered in the dialogues where participants have not discovered yet that the two maps are different, and it is typically produced by IFs when receiving instructions 
for drawing the route which are not compatible with presence and position of landmarks on her/his own map. Interestingly, even though the use of backchannels normally implies signalling understanding and agreement, in these cases a falling contour seems to convey disagreement (or uncertainty) about what is assumed by the interlocutor to be the currently shared background knowledge.
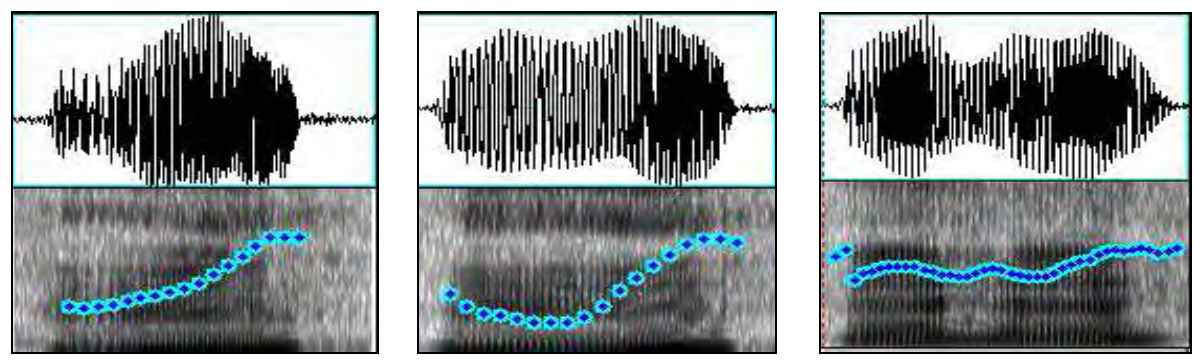

Figure 1. Intonational realisations of ' $\mathrm{mm}$ ' continuers marking PR produced by the same speaker: rising (left), falling-rising (middle), stylised rise (right). In the second case the token is realised as bimoraic, in the third as bysillabic.
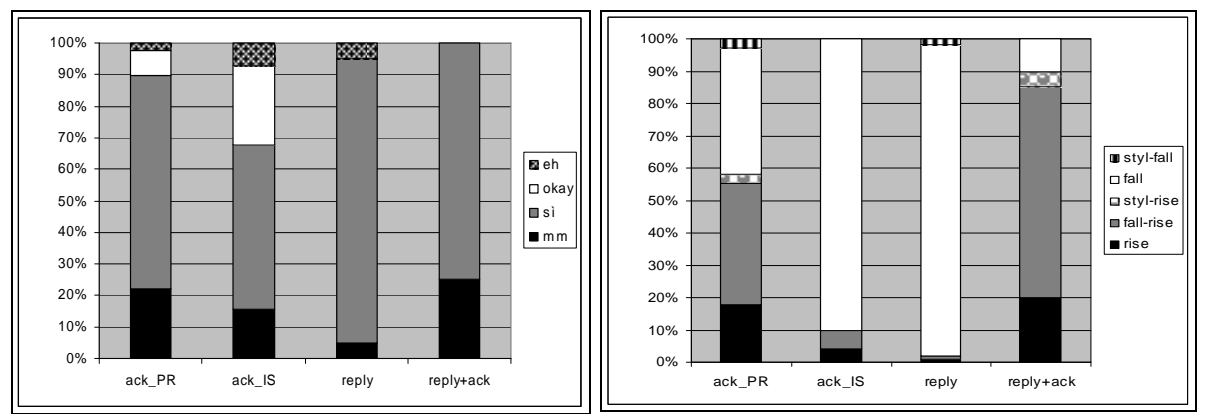

Figure 2. Distribution of token types (left) and F0 contour types (right) as a function of 4 pragmatic classes (acknowledge_Passive-Recipiency, acknowledge_Incipient-Speakership, yes-reply, yes-reply+acknowledge).

Moreover, among backchannels with falling/flat contour, a wide range of phonetic variation (in terms of pitch slope, amplitude, voice quality, duration) is observed (discussion of these cases is not possible here for lack of space). Acknowledge tokens marking IS (i.e. those involving a change of speaker) have predominantly a falling contour, or a "stylised fall" (Figure 2, right panel). Therefore, the intention of taking the floor or not in backchanneling is conveyed intonationally by using a falling vs a rising contour. In general, acknowledge tokens marking IS are not distinguishable from the corresponding tokens used in yes-replies (Figure 2, right panel), as they both share the same F0 contour. However, especially in positive 
answers, there was a great deal of phonetic variation conveying added paralinguistic meanings like surprise, involvement or uncertainty. There was also a number of cases involving a "mixed" pragmatic strategy for conveying yes-answering and backchanneling simultaneously. Here, tokens produced for replying to yes-no questions are intonationally realised with the same contours typically used in continuers marking PR, namely fall-rise, rise or stylised rise (Figure 2, right panel).

\section{Conclusions}

This paper presents an investigation on intonational strategies for backchanneling in Bari Italian task-oriented dialogues. Results show that whilst giving feedback, listeners indicate their intention to take the floor or not by using a falling or rising intonation contour. However, this general tendency can be reversed if added paralinguistic meanings are conveyed.

\section{References}

Benus, S., Gravano, A., Hirschberg, J. 2007. The prosody of backchannels in American English. In Proc. of ICPhS 2007, 1065-10-68, Saarbruecken, Germany.

Carletta, J., Isard, A., Isard, S., Kowtko, J., Doherty-Sneddon, G., Anderson, A. 1997. The Reliability of a Dialogue Structure Coding Scheme. Computational Linguistics 23 (1), 13-32.

Caspers, J. 2000. Melodic characteristics of backchannels in Dutch Map Task dialogues. In Proc. of ICSLP 2000, Bejiing, China.

Cerrato, L., D'Imperio, M. 2003. Duration and tonal characteristics of short expressions in Italian. In Proc. of ICPhS 2003, 1213-1216, Barcelona, Spain.

Grice, M., Savino, M. 2003. Map Tasks in Italian: asking questions about given, accessible and new information. Catalan Journal of Linguistics 2,153-180.

Jefferson, G. 1983. Notes on Systematic Deployment of the Acknowledgment Tokens "yeah" and "mm hm". TILL, Tilburg University, The Netherlands.

Jurafsky, D., Shriberg E., Fox, B., Curl, T. 1998. Lexical, Prosodic, and Syntactic Cues for Dialogue Acts. In Proc. ACL/COLING 98 workshop on Discourse Relations and Discourse Markers, 114-120.

Savino, M. 2001. Non-finality and pre-finality in Bari Italian intonation: a preliminary account. In Proc. of Eurospeech 2001, 939-942, Aalborg, Denmark.

Savino, M. 2004. Intonational cues to discourse structure in a variety of Italian. In Gilles, P., Peters, J. (eds.), Regional variation in intonation, 145-159, Tuebingen: Niemeyer.

Schegloff, E. 1982. Discourse as an interactional achievement: some uses of 'uh huh' and other things that come between sentences. In Tannen, D. (ed.), Analysing Discourse, 71-93. Washington DC, Georgetown Univ. Press.

Ward, N. 2004. Pragmatic functions of prosodic features in non-lexical utterances. In Proc. of Speech Prosody 2004, Nara, Japan. 